STRUCTURAL BIOLOGY COMMUNICATIONS

ISSN 2053-230X

\section{Cloning, expression, purification and crystallization of a pair of novel virulence factors, SghA and SghR, from Agrobacterium tumefaciens}

\author{
Fuzhou Ye, ${ }^{a}$ Chao Wang, ${ }^{b, c}$ Qinqin Fu, ${ }^{a}$ Lian-hui Zhang ${ }^{\mathrm{b}}$ and Yong-gui Gao ${ }^{\mathrm{a}, \mathrm{b} *}$

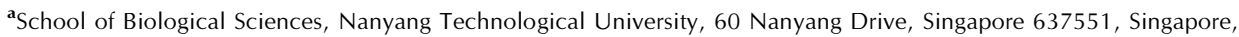 \\ ${ }^{\mathbf{b}}$ Institute of Molecular and Cell Biology, 61 Biopolis Drive, Singapore 138673, Singapore, and ${ }^{\mathbf{c}}$ Division of Cellular and \\ Molecular Research, National Cancer Centre Singapore, 11 Hospital Drive, Singapore 169610, Singapore. \\ *Correspondence e-mail: ygao@ntu.edu.sg
}

Edited by T. C. Terwilliger, Los Alamos National Laboratory, USA

Keywords: SghA; SghR; crystallization; virulence factor; Agrobacterium; plant-microbe interaction; salicylic acid; lacl; chemical signal; glucosidase.

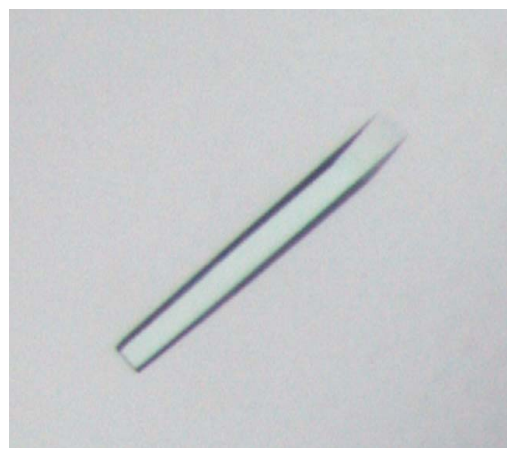

Two proteins, SghA and SghR, which were recently identified and characterized as novel bacterial virulence factors regulating the infection of plant hosts by Agrobacterium, were cloned, overexpressed and purified with high yield. Both SghA and SghR form dimers in solution. The purified SghA and SghR were crystallized and the crystals diffracted to 1.9 and $2.1 \AA$ resolution, respectively. Data were collected and processed, and the crystallographic parameters were within acceptable ranges. These results will help in the determination of their structures in order to uncover the molecular mechanism of how these two proteins together control the release of plant defence signals against agrobacteria during pathogen-host interaction.

\section{Introduction}

The mechanisms of infection of their hosts by bacteria have been widely reported. However, how bacteria strategically control the biosynthesis of the infection machinery and the timely shutting off of the energy-consuming infection process once infection has been successfully established are largely unknown. Agrobacterium tumefaciens has been reported to be the causative agent of crown gall disease (the formation of plant tumours) in over 140 plant species, making it of great concern to the agricultural industry (Moore et al., 1997). Agrobacteria start infection via integrating the oncogenic T-DNA (transferred DNA) from the bacterial tumourinducing (Ti) plasmid into the genome of plant hosts, which also renders this bacterial pathogen a powerful tool for plant genetic modification (Gelvin, 2003; Tzfira \& Citovsky, 2006). In our research, A. tumefaciens was chosen as an experimental model to decipher host-pathogen interaction. Infection by A. tumefaciens involves a number of virulence factors and concomitant plant-derived chemical signals such as salicylic acid (SA), indole-3-acetic acid (IAA) and quorum-sensing (QS) signal (Baron \& Zambryski, 1995; Chevrot et al., 2006; Yuan et al., 2007; Zhang et al., 2002; Liu \& Nester, 2006). It has been reported that these signals play different roles in finetuning the responses of plants and bacteria at different time points during infection (Klessig et al., 2000; Subramoni et al., 2014).

SA is a well characterized plant defence signal in response to different pathogens (Delaney et al., 1994; Gaffney et al., 
Table 1

Macromolecule-production information for SghA.

\begin{tabular}{ll}
\hline Source organism & $\begin{array}{l}\text { A. tumefaciens } \\
\text { DNA source }\end{array}$ \\
Forward primer $\dagger$ & CCGCTCGAGATGGATGACGAAAGGGC \\
Reverse primer $\dagger$ & CCGCTCGAGAAAGCCTCACCCCTTC \\
Cloning vector & pET-14b \\
Expression vector & pET-14b \\
Expression host & E. coli BL21 CodonPlus(DE3) RIL \\
Complete amino-acid sequence & MGSSHHHHHSSGLVPRGSHMLEMDDERAYPMTD- \\
\cline { 2 - 2 } of the construct produced $\$$ & HKALAARFPGDLFGVATASFQIEGATKVDGR- \\
& KPSIWDAFCNMPGHVFGHNGDVACDHYNRW- \\
& DDLDLIKEMGVEAYRFSIAWPRIIPDGFGPIN- \\
& EKGLDFYDRLVDGCKARGIKTYATLYHWDLPL- \\
& TLMGDGGWASRSTAHAFQRYAKTVMARLGDRL- \\
& DAVATFNEPWCAVWLSHLYGIHAPGERNMEAA- \\
& LAAMHHINLAHGFGVEASRHVAPKVPVGLVLN- \\
& AHSVIPASNSDADMKAAERAFQFHNGAFFDPV- \\
& FKGEYPAEMIEALGSRMPVVEAEDLSIISQKL- \\
& DWWGLNYYTPMVADDATEGAEFPATKQAPAV- \\
& SDVKTDIGWEVYAPALHSLVETLYERYELPDC- \\
& YITENGACYNMGVENGEVDDQPRLDYYAEHLG- \\
& IVADLVKDGYPMRGYFAWSLMDNFEWAEGYRM- \\
& RFGLVHVDYETQVRTLKNSGKWYSALASGFPK- \\
& GNHGVMKG \\
\hline
\end{tabular}

$\dagger$ XhoI restriction sites are underlined. $\ddagger$ The extra amino acids introduced into the wild-type SghA protein by cloning are underlined. The primary sequence of the SghA protein listed here corresponds to that reported by Henkel et al. (2014).

1993; Zottini et al., 2007; Anand et al., 2008). During Agrobacterium infection, SA can inhibit the expression of the vir genes and bacterial growth (Yuan et al., 2007; Gohlke \& Deeken, 2014). Unlike other plant-derived chemical signals (such as IAA and cytokinin), whose biosynthetic genes are carried in T-DNA (thus, the expression of T-DNA promotes the de novo synthesis of IAA and cytokinin in plant hosts), no genes are found in T-DNA for the biosynthesis of SA (Akiyoshi et al., 1987; Hwang et al., 2010; Liu et al., 1982). In contrast, the timely release of SA takes place by enzymatic hydrolysis of its storage conjugated form SA $2-O-\beta$-D-glucoside (SAG). Evidence has demonstrated that the concentration of SA is low at the beginning of bacterial infection, but dramatically increases along with a stronger and stronger systematically acquired resistance at the late stage of Agrobacterium infection (Albert, 2013; Gohlke \& Deeken, 2014; Lee et al., 2009). The accumulation of endogenous SA at the late stage is in turn closely correlated with T-DNA integration and vir gene expression (Albert, 2013; Ditt et al., 2001; Veena et al., 2003). However, how the timely regulation of the SA level during the bacterial infection takes place remains elusive.

Our unpublished results identified that a pair of novel proteins, SghA and SghR, from A. tumefaciens A6 are responsible for the temporal regulation of SA concentration in plants during Agrobacterium infection, which is independent of the typical VirA/VirG signalling pathway (Stachel \& Zambryski, 1986). Sequence analyses revealed that SghA belongs to glucosidase hydrolase family 1 . A $B L A S T$ search of the PDB found several homologue structures, the top two among which are BcBgl from Bacillus circulans subsp. alkalophilus (PDB entry 1qox; Hakulinen et al., 2000) and Tmbgl from Thermotoga maritima (PDB entry 1od0; Zechel et al., 2003). The sequence identities of these two proteins to SghA
Table 2

Macromolecule-production information for SghR.

\begin{tabular}{ll}
\hline Source organism & A. tumefaciens \\
DNA source & Genomic DNA \\
Forward primer $\dagger$ & CCGCTCGAGATGAACGATACTGGTAATTCCG \\
Reverse primer $\dagger$ & CCGCTCGAGGCGTTCCTTCTATCAAGG \\
Cloning vector & pET-14b \\
Expression vector & pET-14b \\
Expression host & E. coli BL21 CodonPlus(DE3) RIL \\
Complete amino-acid sequence & MGSSHHHHHSSGLVPRGSHMNTGNSGRDEAKA- \\
\cline { 2 - 2 } of the construct produced $¥$ & TTGERPTLKTIAYMTGLGITTVSRALKDAPDI- \\
& GAETKERVRLIAQQIGYQPNRAGVRLRTGKTN- \\
& VIALVLSVDEELMGFTSQMVFGITEVLATTQY- \\
& HLVVTPHTHAKDSMVPIRYILETGSADGVIIS- \\
& KIEPNDPRVRFMTERKMPFVTHGRSDMGIEHA- \\
& YHDFDNEAYAYEAVERLAQCGRKRIAIIVPPS- \\
& RFAFHDHARKGFTRGIRDFGVSEFPLDAITIE- \\
& TPLDKIRDFGKRLMQSDDRPDGIVSISGSSTI- \\
& ALVAGFEAAGVRIGKDIDIVSKQSAEFLNWIQ- \\
& PQIHTVNEDIKLAGRELAKALLARINGAPPET- \\
& LQSVSRPVWSSMAPKP \\
\hline
\end{tabular}

$\dagger$ XhoI restriction sites are underlined. $\ddagger$ The extra amino acids introduced into the wild-type SghR protein by cloning are underlined. The primary sequence of the SghA protein listed here corresponds to that reported by Henkel et al. (2014).

are 45.0 and $43.0 \%$, respectively. Our functional studies demonstrate that SghA specifically hydrolyzes the inactive SAG to release the active SA, which subsequently inhibits the expression of other vir genes (VirA, VirD2, VirE2 etc.), ultimately avoiding the energy-consuming process of biosynthesizing the infection machinery. This strategy helps Agrobacterium colonization and saves energy for spreading the infection in a self-controlled mode. Furthermore, we identified a transcription factor SghR in A. tumefaciens A6, a homologue of Atu1522 from $A$. tumefaciens $\mathrm{C} 58$, that negatively regulates the transcription of $\operatorname{sgh} A$ at an early stage of bacterial infection via physically binding to its promoter region. SghR assembles as a member of the lacI family of transcription factors containing an N-terminal DNA-binding domain and a C-terminal regulatory domain (Bell \& Lewis, 2000; Lewis et al., 1996; Lewis, 2005). Sequence alignment of SghR with the reported lacI family member Atu1522 (PDB entry 3gv0; New York SGX Research Center for Structural Genomics, unpublished work) from A. fabrum strain C58 and Cagg_2268 (PDB entry 3bbl; New York SGX Research Center for Structural Genomics. unpublished work) gave 91.2 and $25.7 \%$ identity, respectively. However, the Atu1522 structure did not contain the N-terminal DNA-binding domain. Experiments have indicated that both SghA and SghR control tumour growth during Agrobacterium infection and SghA plays a role in the late stage when the infection has been successfully established. Here, we report our preliminary data, including cloning, expression, purification, crystallization and data collection, on these two novel virulence factors.

\section{Materials and methods}

2.1. Cloning, expression and purification of $\operatorname{SghA}$ and $\mathrm{SghR}$

Genes encoding SghA and SghR from A. tumefaciens A6 were amplified by PCR using the primers 5'-CCGCTCGA- 
Table 3

Crystallization of SghA and SghR.

\begin{tabular}{|c|c|c|}
\hline Protein & $\operatorname{Sgh} A$ & SghR \\
\hline Method & Vapour diffusion & Vapour diffusion \\
\hline Plate type & 24-well hanging drop & 24-well hanging drop \\
\hline Temperature $(\mathrm{K})$ & 293 & 293 \\
\hline Protein concentration $\left(\mathrm{mg} \mathrm{ml}^{-1}\right)$ & 4 & 6.4 \\
\hline Buffer composition of protein solution & $50 \mathrm{~m} M$ Tris- $\mathrm{HCl} \mathrm{pH} 7.5,50 \mathrm{~m} M \mathrm{NaCl}, 1 \mathrm{~m} M$ TCEP & $50 \mathrm{~m} M$ HEPES pH 7.0, $50 \mathrm{~m} M \mathrm{NaCl}, 2 \mathrm{~m} M$ TCEP \\
\hline Composition of reservoir solution & $20 \%(w / v)$ PEG $3350,0.4 \mathrm{M}$ ammonium formate & $0.05 M$ HEPES Na pH 7.0, 20\% PEG 3350, $1 \%(w / v)$ tryptone \\
\hline Volume and ratio of drop $\dagger$ & $2 \mu \mathrm{l}, 1: 1$ & 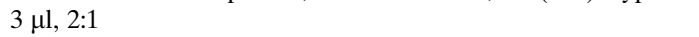 \\
\hline Volume of reservoir $(\mu \mathrm{l})$ & 400 & 400 \\
\hline
\end{tabular}

$\dagger$ The volume ratio is that of protein:reservoir.

GATGGATGACGAAAGGGC-3' (forward) and 5'-CCGCTCGAGAAAGCCTCACCCCTTC-3' (reverse) for SghA and 5'-CCGCTCGAGATGAACGATACTGGTAATTCCG$3^{\prime}$ (forward) and 5'-CCGCTCGAGGCGTTCCTTCTATCAAGG-3' (reverse) for SghR using A. tumefaciens A6 genomic DNA as the PCR template. Detailed molecular cloning information for SghA and SghR is listed in Tables 1 and 2, respectively. The amplified fragments were inserted into the expression vector $\mathrm{pET}-14 \mathrm{~b}$. The recombinant plasmids were verified by DNA sequencing and then transformed into Escherichia coli BL21 CodonPlus(DE3) RIL cells for protein expression.

For large-scale expression of SghA protein, the E. coli BL21 cells were cultured in $2 \times$ YT medium with antibiotics $\left(100 \mu \mathrm{g} \mathrm{ml}^{-1}\right.$ ampicillin and $34 \mu \mathrm{g} \mathrm{ml}^{-1}$ chloramphenicol) at $37^{\circ} \mathrm{C}$. When the optical density $\left(\mathrm{OD}_{600}\right)$ of the cell cultures reached $\sim 0.8$, protein expression was induced by adding $0.5 \mathrm{~m} M$ isopropyl $\beta$-D-1-thiogalactopyranoside (IPTG) at $16^{\circ} \mathrm{C}$. After $18 \mathrm{~h}$ of induction, the cells were harvested by centrifugation $\left(5000 \mathrm{rev} \min ^{-1}, 30 \mathrm{~min}, 4^{\circ} \mathrm{C}\right.$ ). The cell pellets were resuspended in lysis buffer $[50 \mathrm{~m} M$ Tris- $\mathrm{HCl} \mathrm{pH} 7.5$, $150 \mathrm{~m} M \mathrm{NaCl}, 20 \mathrm{~m} M$ imidazole, $5 \mathrm{~m} M \beta$-mercaptoethanol $(\beta$-ME)]. The cell suspension was lysed with a Panda disruptor (GEA Niro Soavi, Italy) and clarified by centrifugation (22 $000 \mathrm{rev} \mathrm{min}^{-1}, 20 \mathrm{~min}, 4^{\circ} \mathrm{C}$ ). The supernatant was collected and filtered through a $0.45 \mu \mathrm{m}$ Minisart filter unit (Sartorius Biotech). Subsequently, the filtered supernatant was loaded onto a $5 \mathrm{ml} \mathrm{Ni-NTA} \mathrm{column} \mathrm{(GE} \mathrm{Healthcare)} \mathrm{and}$ eluted with a linear gradient increase of imidazole concentration $(0.02-0.5 M)$. After SDS-PAGE analysis, fractions containing the target proteins were pooled together and dialyzed against $50 \mathrm{~m} M$ Tris- $\mathrm{HCl} \mathrm{pH} \mathrm{7.5,} 50 \mathrm{~m} M \mathrm{NaCl}, 5 \mathrm{~m} M$ $\beta$-ME). Samples were further purified by anion-exchange chromatography with a HiTrap Q HP Column (GE Healthcare). Elution was conducted with a linear gradient of $\mathrm{NaCl}$ concentration $(0.05-1 M)$ and analyzed by SDS-PAGE. The target proteins were then pooled together, concentrated and loaded onto a HiLoad Superdex 200 26/60 gel-filtration column pre-equilibrated with buffer consisting of $50 \mathrm{mM}$ Tris$\mathrm{HCl} \mathrm{pH} 7.5,50 \mathrm{mM} \mathrm{NaCl}, 1 \mathrm{~m} M$ tris(2-carboxyethyl)phosphine (TCEP). After column elution and checking by SDSPAGE, the target proteins were collected, concentrated to $17 \mathrm{mg} \mathrm{ml}^{-1}$, flash-frozen in liquid nitrogen and stored at $-80^{\circ} \mathrm{C}$.
For SghR protein preparation, the bacterial cells were harvested using a protocol similar to that for SghA. The cells

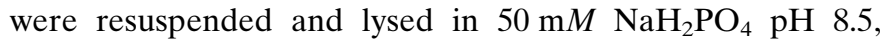
$300 \mathrm{~m} M \mathrm{NaCl}, 5 \mathrm{~m} M \quad \beta$-ME and clarified by centrifugation (22 $000 \mathrm{rev} \mathrm{min}^{-1}, 30 \mathrm{~min}, 4^{\circ} \mathrm{C}$ ). The sample was first loaded onto an Ni-NTA affinity column (GE Healthcare) and then eluted with a linear gradient increase of imidazole concentration $(0-0.5 M)$. After checking by SDS-PAGE, the fractions containing the target protein were pooled, concentrated and loaded onto a HiLoad Supderdex 200 26/60 gel-filtration column pre-equilibrated with $50 \mathrm{~m} M$ HEPES $\mathrm{pH} 7.0,50 \mathrm{~m} M$ $\mathrm{NaCl}, 2 \mathrm{~m} M$ TCEP. The target protein was eluted, concentrated to $6.4 \mathrm{mg} \mathrm{ml}^{-1}$, flash-frozen in liquid nitrogen and stored at $-80^{\circ} \mathrm{C}$ for subsequent experiments.

\subsection{Molecular-weight calibration of SghA and SghR}

To calculate the molecular weights of SghA and SghR in solution, the High Molecular Weight (HMW) gel-filtration calibration kit (GE Healthcare) was used. The gel-filtration column (Superdex 200, 10/300 GL) was first equilibrated with the sample buffer (50 $\mathrm{m} M$ HEPES pH 7.0, $50 \mathrm{~m} M \mathrm{NaCl}, 2 \mathrm{~m} M$ TCEP). Blue dextran 2000 (to determine the void volume), a mixture of four standard proteins (ovalbumin, $44000 \mathrm{Da}$; conalbumin, $75000 \mathrm{Da}$; aldolase 2, $158000 \mathrm{Da}$; ferritin 2, $440000 \mathrm{Da}), \mathrm{Sgh} A$ and SghR were sequentially loaded onto the column in four separate runs. Following the instructions for the kit, the $K_{\mathrm{av}}$ was plotted against $\log$ (molecular weight). Consequently, the molecular weights of SghA and SghR in solution were determined.

\subsection{Crystallization and data collection}

Crystallization screening of SghA (diluted to $4 \mathrm{mg} \mathrm{ml}^{-1}$ ) and $\mathrm{SghR}$ (at $6.4 \mathrm{mg} \mathrm{ml}^{-1}$ ) was performed. For each condition, three varied ratios of protein and reservoir solution $(0.2: 0.1 \mu \mathrm{l}$, 0.15:0.15 $\mu \mathrm{l}$ and 0.1:0.2 $\mu \mathrm{l}$ ) were screened by the sitting-drop vapour-diffusion method using a Phoenix robot (Art Robbins Instruments) at $20^{\circ} \mathrm{C}$. Screening kits from Hampton Research, including Crystal Screen, Crystal Screen 2, Index, PEG/Ion, PEGRx and SaltRx, were used. Detailed information on SghA and SghR crystallization is listed in Table 3.

For SghA, initial crystals were obtained in a condition consisting of $20 \%(w / v)$ PEG 3350, $0.4 M$ ammonium formate using $0.1 \mu \mathrm{l}$ protein solution and $0.1 \mu \mathrm{l}$ reservoir solution. 
Table 4

Data-collection and processing statistics.

Values in parentheses are for the outer shell.

\begin{tabular}{|c|c|c|}
\hline Data set & $\operatorname{Sgh} A$ & SghR \\
\hline Diffraction source & I04, DLS & I04, DLS \\
\hline Wavelength $(\AA)$ & 0.9795 & 0.9795 \\
\hline Temperature (K) & 100 & 100 \\
\hline Crystal-to-detector distance (mm) & 245 & 287.7 \\
\hline Rotation range per image $\left({ }^{\circ}\right)$ & 0.2 & 0.4 \\
\hline Total rotation range $\left({ }^{\circ}\right)$ & 90 & 90 \\
\hline Exposure time per image (s) & 1 & 1 \\
\hline Space group & $P 2{ }_{1} 2_{1} 2_{1}$ & $P 2{ }_{1} 2_{1} 2_{1}$ \\
\hline Unit-cell parameters $\left(\AA,^{\circ}\right)$ & $\begin{array}{l}a=64.2, b=80.4 \\
\quad c=184.62 \\
\alpha=\beta=\gamma=90\end{array}$ & $\begin{array}{l}a=35.54, \\
b=119.45, \\
c=121.94, \\
\alpha=\beta=\gamma=90\end{array}$ \\
\hline Average mosaicity $\left({ }^{\circ}\right)$ & 0.26 & 0.32 \\
\hline Resolution range $(\AA)$ & $50-1.9(2.10-1.90)$ & $50-2.1(2.21-2.10)$ \\
\hline Total No. of reflections & 263299 & 110508 \\
\hline No. of unique reflections & 73481 & 31202 \\
\hline Completeness (\%) & $99.7(100)$ & 99.7 (99.9) \\
\hline Multiplicity & $3.4(3.4)$ & $3.5(3.6)$ \\
\hline$\langle I / \sigma(I)\rangle$ & $8.8(2.5)$ & $9.6(3.7)$ \\
\hline$R_{\text {merge }}(\%)$ & $10.0(47.1)$ & $8(32.7)$ \\
\hline $\begin{array}{l}\text { Overall } B \text { factor from Wilson } \\
\quad \text { plot }\left(\AA^{2}\right)\end{array}$ & 18.5 & 23.1 \\
\hline
\end{tabular}

Further optimization by fine-tuning the PEG concentration (19-21\%) and increasing the drop size to $1 \mu$ protein solution and $1 \mu \mathrm{l}$ reservoir solution was carried out applying both the hanging-drop and the sitting-drop methods. Only the hangingdrop method gave decent crystals. We did not obtain any crystals by the sitting-drop method as most wells precipitated. After the crystals reached maximum size $(10 \mathrm{~d})$, they were cryoprotected in crystallization buffer containing 35\% PEG 3350 before flash-cooling in liquid nitrogen.
For SghR, initial crystal hits were observed in a condition consisting of $0.05 M$ HEPES Na pH 7.0, 20\% PEG 3350, $1 \%(w / v)$ tryptone using $0.2 \mu \mathrm{l}$ protein solution and $0.1 \mu \mathrm{l}$ reservoir solution. As the tryptone in the crystallization condition is a bacterial nutrient, the drop is easily contaminated by bacteria, which interfere with the crystallization process. To avoid bacterial contamination, all reagents used for SghR optimization were filtered through a $0.2 \mu \mathrm{l}$ filter and the optimization plates were set up in a fume cupboard. The crystallization condition was optimized by varying the PEG concentration (17-22\%) and increasing the drop size to $2 \mu \mathrm{l}$ protein solution and $1 \mu \mathrm{l}$ reservoir solution. The crystals were cryoprotected with a cryoprotectant consisting of $35 \%$ PEG 3350 in the reservoir and were then flash-cooled in liquid nitrogen.

Diffraction-quality data sets for both SghA and SghR were collected at $100 \mathrm{~K}$ on beamline I04 at Diamond Light Source (DLS) and the data were processed using XDS (Kabsch, 2010). Detailed information on data collection is given in Table 4.

\section{Results and discussion}

The recombinant plasmids pET-14b-SghA and pET-14b-SghR encode the corresponding target proteins with an N-terminal $\mathrm{His}_{6}$ tag. Purification of SghA included three chromatographic steps (sequentially, $\mathrm{Ni}^{2+}$-affinity, anion-exchange and gelfiltration chromatography). In contrast to SghA, two chromatographic steps were adopted for the purification of SghR: $\mathrm{Ni}^{2+}$-affinity and gel-filtration chromatography. The purified SghA and SghR proteins both displayed high purity (Figs. $1 a$ and $1 b$ ), with a final yield of approximately $50 \mathrm{mg}$ protein per

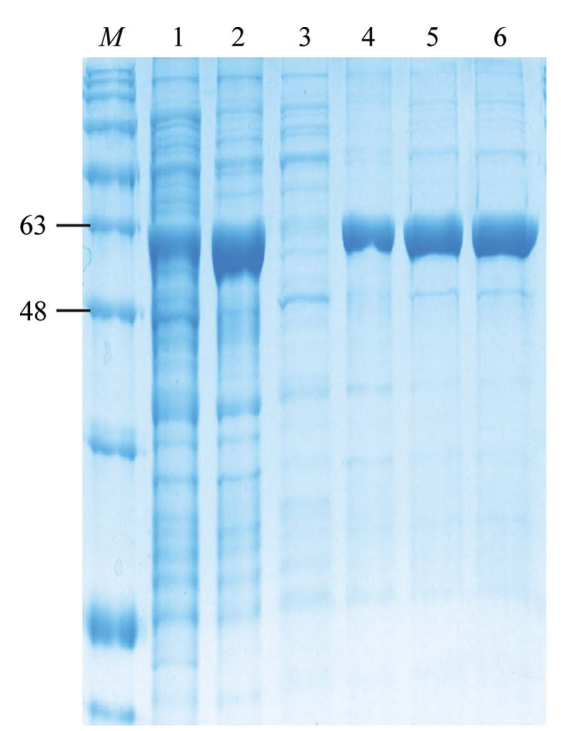

$(a)$

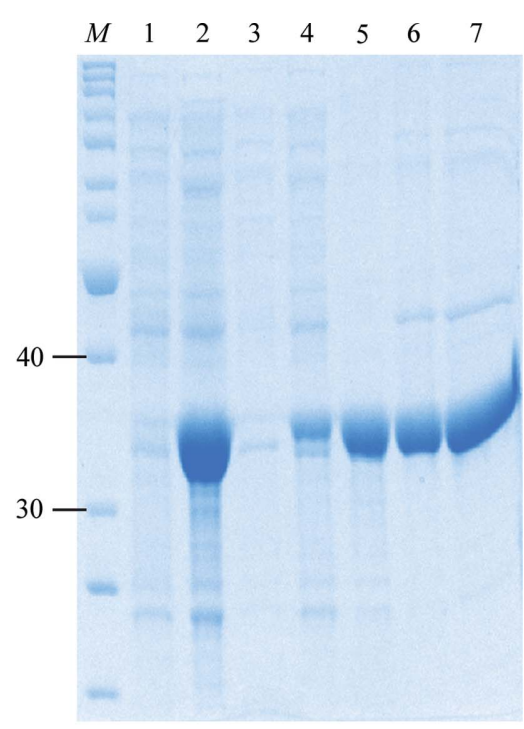

(b)

Figure 1

Expression and purification of SghA $(a)$ and SghR $(b)$. (a) Lane $M$, molecular-weight marker (labelled in kDa); lanes 1 and 2, supernatant and pellet after cell extraction, respectively; lane 3, flowthrough after Ni-NTA affinity binding; lanes 4 and 5, after elution from Ni-NTA affinity column; lane 6, purified SghA protein after gel-filtration chromatography. (b) Lane $M$, molecular-weight marker (labelled in $\mathrm{kDa}$ ); lanes 1 and 2, total cells before and after IPTG induction, respectively; lane 3, flowthrough after Ni-NTA affinity binding; lanes 4 and 5, supernatant and pellet after cell extraction, respectively; lane 6, after elution from Ni-NTA purification; lane 7, purified SghR after gel-filtration chromatography. 
litre of culture. It was noted that there was only a subtle difference in lane 6 in Fig. 1(a) and lane 7 in Fig. 1(b) from the corresponding nickel-affinity purification. This result suggested that the proteins were quite pure after nickelaffinity purification and that the subsequent anion-exchange or gel-filtration chromatography was not essentially able to enhance the protein purity. From the gel-filtration chromatography profiles, the size of both proteins also appeared to be double that of their corresponding monomers. To verify this, the molecular weights of SghA and SghR were calibrated using a HiLoad Superdex 200 10/300 gel-filtration column and the High Molecular Weight calibration kit (GE Healthcare). The calibration results demonstrated that both proteins indeed exist as dimers in solution (Fig. 2).

Crystallization screening and further optimization led to decent plate-shaped and rod-shaped crystals of SghA and SghR, respectively (Fig. 3). Both crystals were harvested with thorough washing with their reservoir solutions. Subsequent SDS-PAGE separation and mass-spectral identification were performed, and the results clearly indicated the crystallized macromolecules were the target proteins SghA and SghR, respectively. Therefore, data collection was carried out and the collected data sets were processed to acceptable $R_{\text {merge}}$, completeness and $\langle I / \sigma(I)\rangle$ values in the highest resolution bin

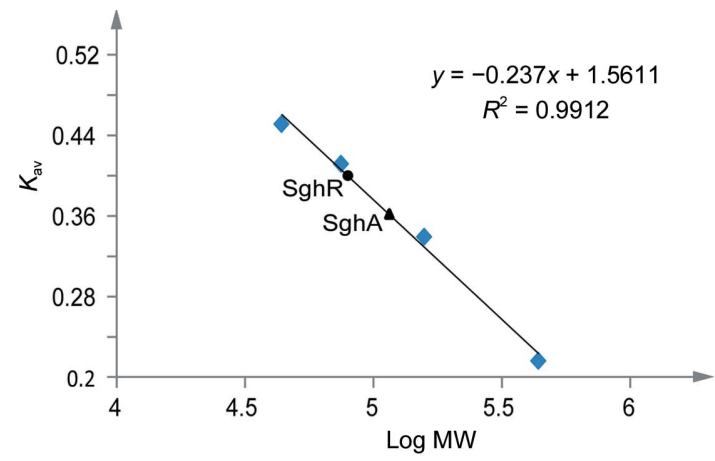

(a)

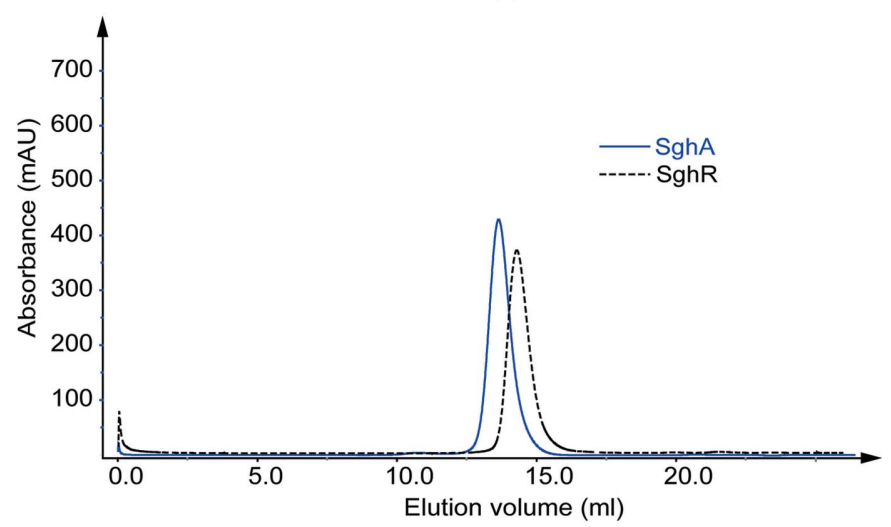

(b)

Figure 2

(a) Standard calibration curve based on the $\log$ (molecular weight) of four different standard proteins plotted against $K_{\mathrm{av}}$. The circle and triangle on the standard curve indicate the positions of SghR and SghA, respectively (depicted based on the $K_{\text {av }}$ value). (b) Gel-filtration chromatography elution profile of SghA (solid line) and SghR (dashed line) used for molecular-weight calibration.
(Fig. 4 and Table 4). Both the SghA and the SghR crystals belonged to space group $P 2_{1} 2_{1} 2_{1}$. The data from the SghA crystals were processed to $1.9 \AA$ resolution, with unit-cell parameters $a=64.2, b=80.4, c=184.62 \AA$, whereas the data from the SghR crystals were processed to $2.1 \AA$ resolution, with unit-cell parameters $a=35.54, b=119.45, c=121.94 \AA$ (Table 1). $V_{\mathrm{M}}$ calculations indicated that there are two molecules in the asymmetric unit. On the basis of our molecularweight calibration results, it appears that the crystals of both proteins contained a dimer in the asymmetric unit. Further model building and structure refinement are ongoing for both structures.

SghA was identified as a novel virulence factor that shuts off the infection machinery through controlling the release of active SA from SAG to inhibit the expression of the other vir genes after infection has been established successfully. Its enzyme specificity toward SAG and its catalytic mechanism will be further investigated by structural study based on the current crystallographic data and on substrate/product-bound forms of SghA together with other enzymatic assays. As a transcriptional repressor, SghR represses the transcription of sghA via physically binding to its promoter region and derepressing the transcription upon sensing the environmental

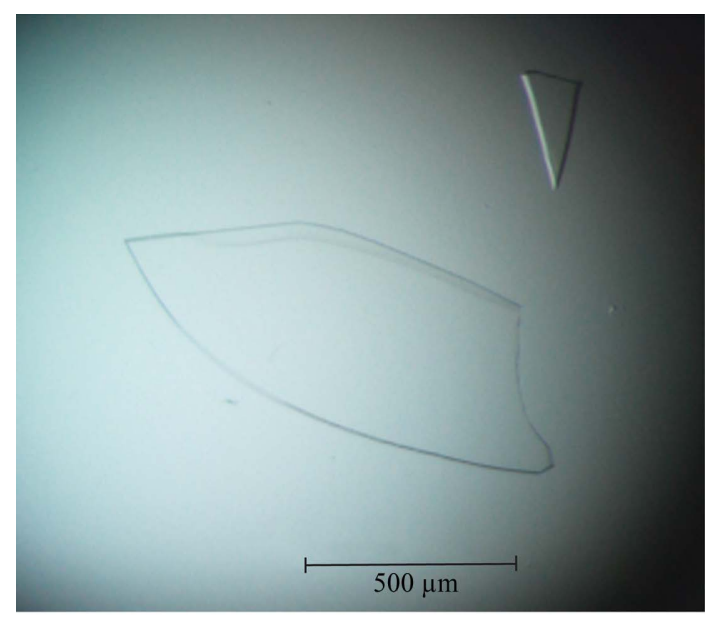

(a)

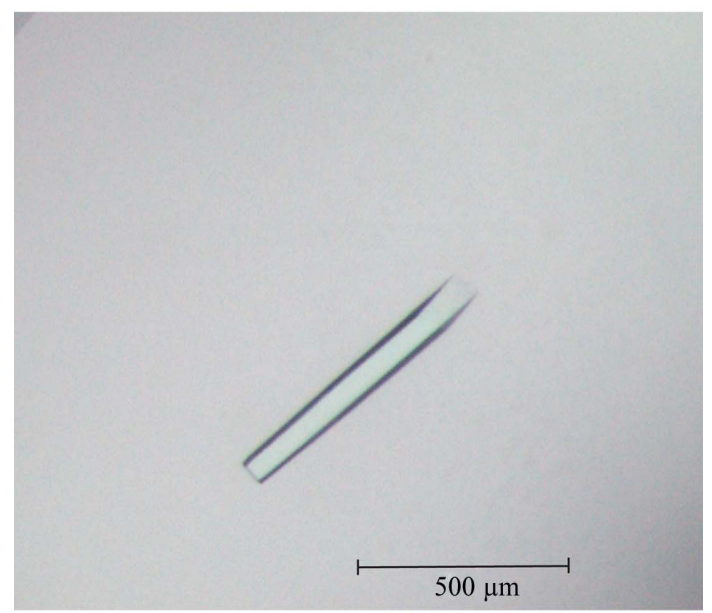

(b)

Figure 3

Crystal photographs of SghA (a) and SghR (b). 


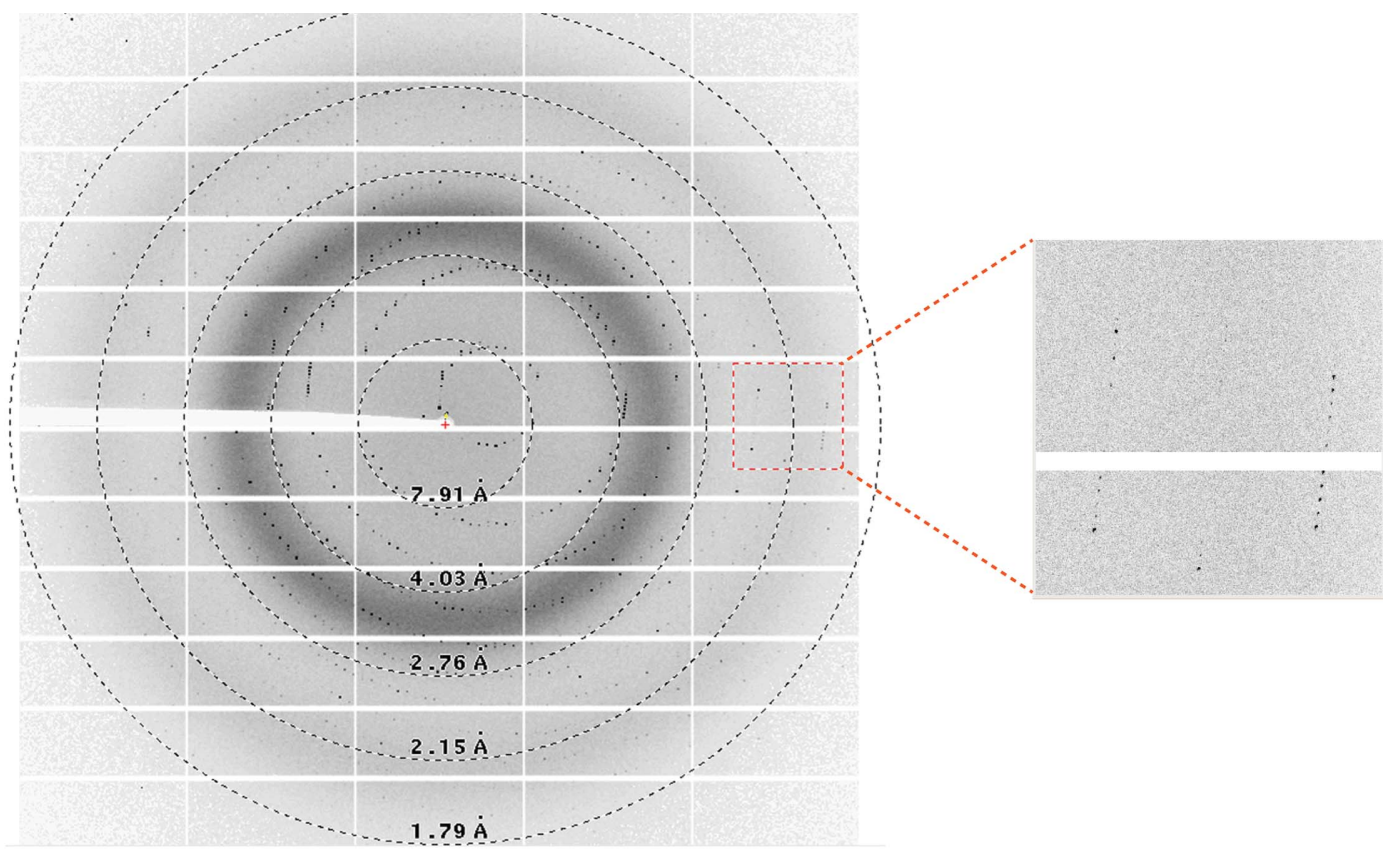

(a)

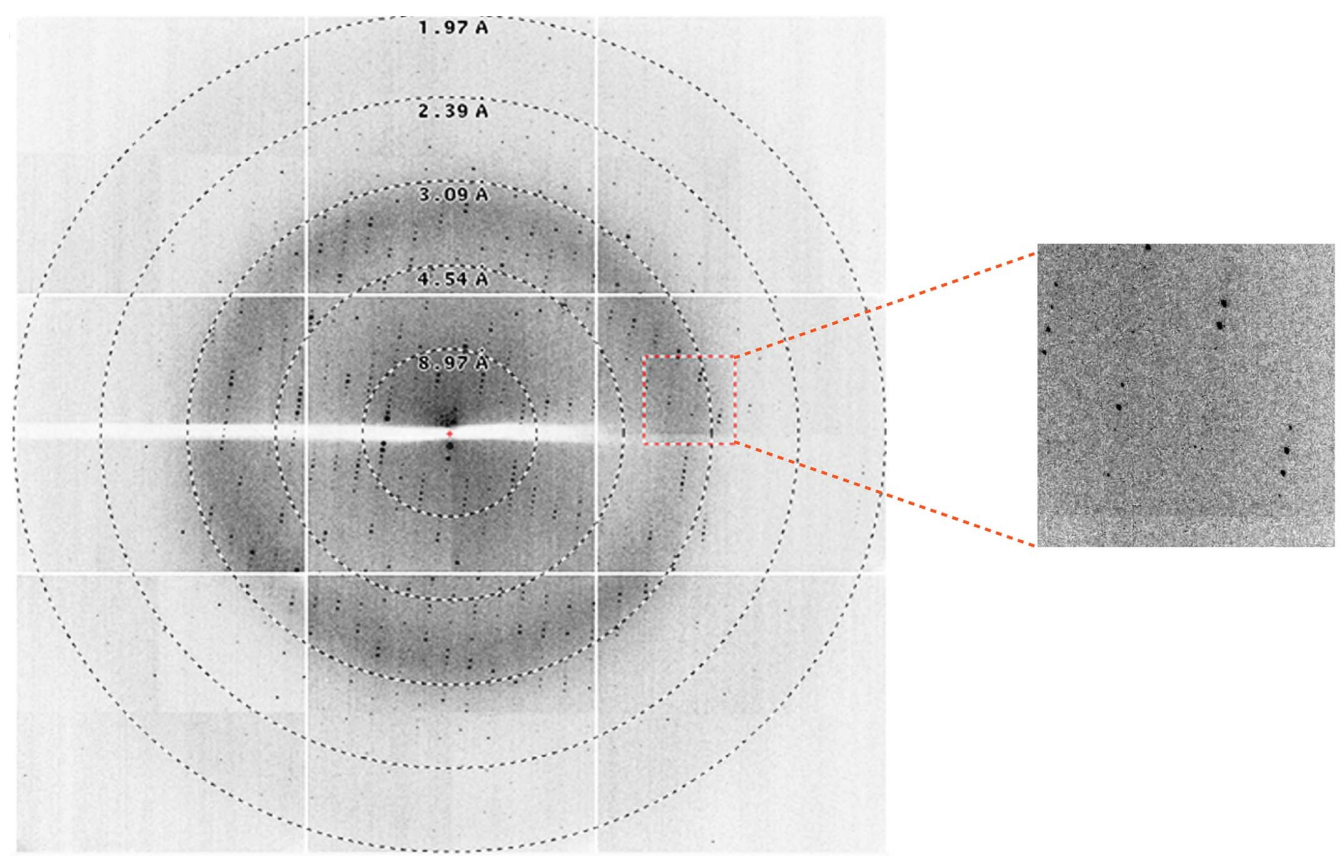

(b)

Figure 4

Diffraction patterns of $\operatorname{SghA}(a)$ and $\operatorname{SghR}(b)$. The right panel is a magnification of the boxed region. The resolution rings were generated using $A D X V$ (http://www.scripps.edu/ arvai/adxv.html).

stimulus. Structural insights into the regulation mechanism of SghA by SghR through the determination of SghR-effector and SghR-DNA complexes are also under way.

\section{Acknowledgements}

The authors declare that they have no competing interests. The authors thank the staff of beamline I04 at Diamond Light Source, UK for assistance during data collection.

\section{References}

Akiyoshi, D. E., Regier, D. A. \& Gordon, M. P. (1987). J. Bacteriol. 169, 4242-4248.

Albert, M. (2013). J. Exp. Bot. 64, 5269-5279.

Anand, A., Uppalapati, S. R., Ryu, C.-M., Allen, S. N., Kang, L., Tang, Y. \& Mysore, K. S. (2008). Plant Physiol. 146, 703-715.

Baron, C. \& Zambryski, P. C. (1995). Annu. Rev. Genet. 29, 107-129.

Bell, C. E. \& Lewis, M. (2000). Nature Struct. Biol. 7, 209-214.

Chevrot, R., Rosen, R., Haudecoeur, E., Cirou, A., Shelp, B. J., Ron, E. \& Faure, D. (2006). Proc. Natl Acad. Sci. USA, 103, 7460-7464. Delaney, T. P., Uknes, S., Vernooij, B., Friedrich, L., Weymann, K., 
Negrotto, D., Gaffney, T., Gut-Rella, M., Kessmann, H., Ward, E. \& Ryals, J. (1994). Science, 266, 1247-1250.

Ditt, R. F., Nester, E. W. \& Comai, L. (2001). Proc. Natl Acad. Sci. USA, 98, 10954-10959.

Gaffney, T., Friedrich, L., Vernooij, B., Negrotto, D., Nye, G., Uknes, S., Ward, E., Kessmann, H. \& Ryals, J. (1993). Science, 261, 754756.

Gelvin, S. B. (2003). Microbiol. Mol. Biol. Rev. 67, 16-37.

Gohlke, J. \& Deeken, R. (2014). Front. Plant Sci. 5, 1-11.

Hakulinen, N., Paavilainen, S., Korpela, T. \& Rouvinen, J. (2000). J. Struct. Biol. 129, 69-79.

Henkel, C. V., den Dulk-Ras, A., Zhang, X. \& Hooykaas, P. J. (2014). Genome Announc. 2, e00225-14.

Hwang, H.-H., Wang, M.-H., Lee, Y.-L., Tsai, Y.-L., Li, Y.-H., Yang, F.-J., Liao, Y.-C., Lin, S.-K. \& Lai, E.-M. (2010). Mol. Plant Pathol. 11, 677-690.

Kabsch, W. (2010). Acta Cryst. D66, 125-132.

Klessig, D. F., Durner, J., Noad, R., Navarre, D. A., Wendehenne, D., Kumar, D., Zhou, J. M., Shah, J., Zhang, S., Kachroo, P., Trifa, Y., Pontier, D., Lam, E. \& Silva, H. (2000). Proc. Natl Acad. Sci. USA, 97, 8849-8855.

Lee, C.-W., Efetova, M., Engelmann, J. C., Kramell, R., Wasternack, C., Ludwig-Muller, J., Hedrich, R. \& Deeken, R. (2009). Plant Cell, 21, 2948-2962.

Lewis, M. (2005). C. R. Biol. 328, 521-548.
Lewis, M., Chang, G., Horton, N. C., Kercher, M. A., Pace, H. C., Schumacher, M. A., Brennan, R. G. \& Lu, P. (1996). Science, 271, 1247-1254.

Liu, S.-T., Perry, K. L., Schardl, C. L. \& Kado, C. I. (1982). Proc. Natl Acad. Sci. USA, 79, 2812-2816.

Liu, P. \& Nester, E. W. (2006). Proc. Natl Acad. Sci. USA, 103, 46584662.

Moore, L. W., Chilton, W. S. \& Canfield, M. L. (1997). Appl. Environ. Microbiol. 63, 201-207.

Stachel, S. E. \& Zambryski, P. C. (1986). Cell, 46, 325-333.

Subramoni, S., Nathoo, N., Klimov, E. \& Yuan, Z.-C. (2014). Front. Plant Sci. 5, 322.

Tzfira, T. \& Citovsky, V. (2006). Curr. Opin. Biotechnol. 17, 147-154.

Veena, Jiang, H., Doerge, R. W. \& Gelvin, S. B. (2003). Plant J. 35, 219-236.

Yuan, Z.-C., Edlind, M. P., Liu, P., Saenkham, P., Banta, L. M., Wise, A. A., Ronzone, E., Binns, A. N., Kerr, K. \& Nester, E. W. (2007). Proc. Natl Acad. Sci. USA, 104, 11790-11795.

Zechel, D. L., Boraston, A. B., Gloster, T., Boraston, C. M., Macdonald, J. M., Tilbrook, D. M., Stick, R. V. \& Davies, G. J. (2003). J. Am. Chem. Soc. 125, 14313-14323.

Zhang, H.-B., Wang, L.-H. \& Zhang, L.-H. (2002). Proc. Natl Acad. Sci. USA, 99, 4638-4643.

Zottini, M., Costa, A., De Michele, R., Ruzzene, M., Carimi, F. \& Lo Schiavo, F. (2007). J. Exp. Bot. 58, 1397-1405. 